\title{
REVISIONES
}

\section{¿Evaluación en competencias?}

Assessment of Skills?

Avaliação por Competência?

\section{Luis Manríquez Pantoja}

Universidad de Antofagasta, Dirección Departamento de Educación, 1manriquez@uantof.cl, 55637368

\section{RESUMEN}

El presente ensayo tiene como fin promover un debate de una cuestión urgente como es el de encontrar un enfoque adecuado para medir o determinar el logro de competencias. Con la debida prevención que ello implica necesariamente adscribirse a un modelo pedagógico coherente con el desarrollo de competencias. Se revisa sucintamente el concepto de evaluación con énfasis en el aprendizaje y a modo de aporte se proponen tres posibles recursos evaluativos para determinar el logro de competencias desarrolladas.

Palabras clave: Competencias, evaluación, mapas conceptuales, rúbricas, portafolios.

\begin{abstract}
This essay aims to promote a debate in an urgent issue as it is to find an appropriate approach to measure or determine the achievement of skills. With proper prevention ascribed, this necessarily implies a pedagogical model consistent with the skills development. We briefly review the concept of assessment with emphasis on learning and three possible assessment resources to determine the achievement of skills developed are presented.
\end{abstract}

Key words: : skill, evaluation, rubrics, mind maps, portfolios.

\section{RESUMO}

Este ensaio objetiva promover um debate acerca de questão urgente, ou seja, como encontrar abordagem adequada para medir ou determinar a conquista de competências. Com a devida prevenção nisso implicada, necessariamente, descreve-se um modelo pedagógico coerente com o desenvolvimento de competências. Revisa-se, brevemente, o conceito de avaliação, com ênfase na aprendizagem e como contribuição propõem-se três recursos avaliativos possíveis para determinar o alcance das competências desenvolvidas.

Palavras chave: competência, avaliação, mapas conceituais, rubricas, portfólios. 


\section{INTRODUCCIÓN}

El concepto de competencia tan propio del mundo laboral, se ha incorporado en el horizonte universitario como un catalizador de modelos curriculares desde la década del 90 asumiendo distintas denominaciones: formación por competencias, planes de estudio basados en el enfoque por competencias, propuestas educativas por competencias; todo con miras a transformarse en una opción alternativa de formación académica.

Hay bastante consenso en justificar esta decisión, lo que puede resumirse principalmente en la característica principal de la época que nos ha correspondido vivir. Una época de cambios exponenciales de todo orden y magnitud, lo que está provocando un desplazamiento del centro de gravedad de la enseñanza universitaria, históricamente encargada de formar profesionales para el mercado laboral, cuya estructura cada vez más se orienta hacia el nivel terciario. Consecuentemente, el contenido de variadas ocupaciones está cambiando rápidamente, generándose nuevas demandas de competencias y conocimientos. Todo ello en el contexto de la globalización y las nuevas tecnologías de la información que evolucionan incesantemente (Brunner, 2003).

Posiblemente con esta perspectiva, en 1998 se inicia un proceso de transformación educativa con la reunión en la Sorbona (Paris) de cuatro ministros de educación superior representantes de Inglaterra, Francia, Italia y Alemania; allí analizan el devenir de la educación superior, promoviendo la necesidad de crear condiciones que favorezcan la movilidad, la cooperación y la convalidación de créditos en la educación inicial y continua. La propuesta de este grupo se traduce en la declaración de Bolonia (Italia) en 1999, con la participación de 30 Estados europeos, lo que el 2000 permite la creación de un proyecto de trabajo colectivo de universidades con el nombre de Proyecto Tuning. Este proyecto, hoy día masificado, evolucionó en Europa a través de distintas etapas, esperándose que al 2010 estuviera compartiendo el mismo Espacio de Educación Superior (Ramírez y Medina, 2008) un número amplio de centros educativos.

Las universidades latinoamericanas, incluidas las de Chile en 2004, cuando Tuning Europa se aprestaba a iniciar su tercera fase, pusieron en marcha un proyecto semejante denominado Tuning Alfa, en la actualidad con la participación de 19 países y casi 200 universidades.

En 2007 la Universidad de Deusto (que con la Universidad de Groningen coordinan el Proyecto Tuning en Europa) publica un libro que da cuenta del Proyecto Tuning en América Latina. De su lectura es posible determinar la diferencia entre ambos proyectos.

En el Tuning Europa, los lineamientos establecidos por «Bolonia» y los compromisos contraídos hacen que el proyecto tenga un marco más preciso. En él participan más de 175 universidades europeas, pero el mismo ritmo del proceso hace que el resto de las instituciones de educación superior se aboquen a discutir, pensar y repensar los resultados alcanzados, cuya meta principal es llegar a compartir, tal como dijimos, un mismo Espacio de Educación Superior, lo que de una manera simplificada debería entenderse que a partir de las competencias consensuadas, exista una gran movilidad de estudiantes y académicos, donde títulos y grados serán válidos en toda la Comunidad Europea desde el concepto de crédito transferible.

En América Latina no se ha fijado una fecha para concretar un Espacio de Educación Superior, tampoco tiene un marco político claramente acordado como el que posee Europa. El proyecto Tuning - América Latina no tiene pretensiones de crear ese Espacio, pero sí 
quisiera brindar elementos que faciliten la reflexión, con el propósito de llegar a acuerdos básicos en el marco de la educación superior. El proyecto se propuso vincular a responsables de la política universitaria de 19 países latinoamericanos para dialogar sobre las potencialidades de colaborar, más allá de las fronteras nacionales (Beneitone et al., 2007).

En el proyecto Tuning - América Latina, además de las universidades, se propuso un segundo actor relevante: los Centros Nacionales Tuning (CNT). Cada país de América Latina participa en el proyecto por igual, a través de estos Centros Nacionales. El espíritu Tuning es que esté abierto a todas las voces, que se incluyan todos los países. Los entes participantes en el proyecto en América Latina. Tuning - América Latina es liderado por las universidades, que son los actores clave en este proceso. Los Centros Nacionales Tuning acompañan a las instituciones en este camino, fortaleciendo en algunos casos este andar e incorporando los aportes de todos los actores que componen su sistema nacional.

En el caso chileno, en cierta medida, las propias autoridades gubernamentales de la época dieron un impulso significativo al Proyecto con la perspectiva de transitar hacia una economía basada en el conocimiento, para disponer de capital humano avanzado suficiente y capaz de llevar adelante investigación e innovación competitiva a nivel mundial, y generar flujos rentables de conocimiento que puedan movilizarse con efectividad en el mundo. Incluido el hecho que en 2006 se promulgó la Ley de Aseguramiento de la Calidad de la Educación Superior. Las actividades del Proyecto Tuning América Latina fue visualizado desde el gobierno como una oportunidad para potenciar dicha renovación y lograr resultados en un menor plazo por lo que aporta recursos a través de los programas denominados MECESUP (Programa de Mejoramiento de la Calidad de la Educación Superior, Ministerio de Educación) para impulsar procesos de renovación curricular.

Según Muñoz y Sobrero (2006), los fines planteados por el Mecesup para la integración de las universidades chilenas a Tuning América Latina coinciden con los que orientan al proyecto europeo y latinoamericano en lo referente a lograr una homologación expedita de estudios universitarios en una forma articulada en toda América Latina en un futuro próximo.

Así, como resultado de las postulaciones efectuadas por las universidades para participar en estos procesos, el Centro Nacional Chile del Proyecto Tuning América Latina -creado en el marco del programa Mecesup- resolvió recomendar dicho proyecto a cinco universidades durante una primera fase iniciada en el 2005, cada una de las cuales desarrolla una o más de las áreas propuestas: Universidad de Chile (Matemáticas), Pontificia Universidad Católica de Chile (Historia), Pontificia Universidad Católica de Valparaíso (Educación), Universidad de Talca (Administración de Empresas), Universidad Metropolitana de Ciencias de la Educación (Historia). Durante una segunda fase de implementación, se integraron ocho universidades más.

Siguiendo la metodología propia, Tuning - América Latina tiene cuatro grandes líneas de trabajo:

1) competencias (genéricas y específicas de las áreas temáticas);

2) enfoques de enseñanza, aprendizaje y evaluación de estas competencias;

3) créditos académicos;

4) calidad de los programas. 
Con la segunda línea, se pretende preparar una serie de materiales que permitan visualizar cuáles serán los métodos de enseñanza, aprendizaje y evaluación más eficaces para el logro de los resultados del aprendizaje y las competencias identificadas. Esto implica desarrollar una combinación novedosa de enfoques de enseñanza y aprendizaje, para estimular —o permitir que se desarrollen — las competencias que se diseñan en el perfil. Los cambios de enfoques y objetivos de enseñanza y aprendizaje implican también modificaciones en los métodos y criterios de evaluación, en función no sólo de los contenidos, sino también de habilidades, destrezas y valores.

Todas las universidades adscritas al Consejo de Rectores y la mayoría de las universidades restantes que iniciaron sus procesos de acreditación de carreras en conformidad a la normativa vigente, de algún modo se involucraron con enfoques relacionados con las competencias, nos imaginamos, con la idea de generar necesarias innovaciones en los enfoques curriculares que ajusten la triada enseñanza, aprendizaje y evaluación; frente a un escenario de una larga tradición de racionalismo académico, transmisión del conocimiento, clases frontales y proceso de certificación (calificación) como sinónimo de evaluación. Posiblemente este proceso podría exacerbarse cuando estas innovaciones afecten a docentes académicos sin formación pedagógica.

La tríada antes mencionada merece un exhaustivo y profundo estudio por conformar los pilares básicos donde transcurre el desarrollo de competencias, esa posibilidad excede los límites del presente trabajo, que en particular pretende reflexionar respecto a uno de estos componentes: la evaluación en el contexto de las competencias.

Previamente deberemos enfrentar la frecuente polisemia de conceptos que ocurre en la Ciencias de la Educación, ¿qué deberíamos entender por competencia? Por ejemplo, Cabrerizo, Rubio y Castillo (2008) aportan 11 definiciones de este término, pero se puede plantear una convencional de la siguiente manera:

Son las capacidades que todo ser humano necesita para resolver, de manera eficaz y autónoma, las situaciones de la vida. Se fundamentan en un saber profundo, no sólo saber qué y saber cómo, sino saber ser persona en un mundo complejo, cambiante y competitivo (Beneitone et al., 2007:35)

\section{UN MODELO PEDAGÓGICO COHERENTE:}

Para avanzar en nuestro estudio es necesario previamente determinar un modelo pedagógico coherente con la filosofía de las competencias; los autores no se han puesto de acuerdo bajo que enfoque paradigmático deberían desarrollarse.

Nosotros abordaremos esta primera problemática aceptando la autoridad del informe de las universidades Deusto-Groningen ya citado; en su calidad de responsables coordinadores del Proyecto Tuning, expresan al respecto:

Otro paradigma de la educación actual es que el centro del proceso de enseñanza- aprendizaje deja de ser el profesor y pasa a ser el estudiante. Es necesario que los docentes cedan el escenario, el protagonismo, la palabra y el tiempo a los estudiantes. De modo que, de la educación centrada en la enseñanza, se pase a aquélla sustentada en el aprendizaje. 
El estudiante pasa a tener una participación activa en la construcción de su propio aprendizaje, con lo que el profesor se convierte en el gran facilitador que pone en manos de los estudiantes los recursos: información, métodos, herramientas, crea ambientes y les acompaña, brindándoles asistencia a lo largo de todo el proceso, elevando con ello su motivación, compromiso y gusto por aprender y comprender la utilidad del aprendizaje. Es necesario que se generalice el profesor motivador, facilitador de procesos de desarrollo humano y asesor científico y metodológico. (Beneitone et al., 2007:25)

Sobre el punto, nos llama la atención que el marco curricular vigente tanto para la Enseñanza Básica y Media chilena, está expresado en términos parecidos (MINEDUC, 2009), como puede constatar al transcribir su parte introductoria.

Los OF-CMO [Objetivos Fundamentales-Contenidos Mínimos Obligatorios] del marco curricular están orientados al desarrollo de competencias que se consideran fundamentales para el desarrollo personal y para desenvolverse en el ámbito social, laboral y ciudadano. Siguiendo una definición de amplio consenso sobre las competencias, éstas aluden a la capacidad para responder a las exigencias individuales o sociales para realizar una tarea o, dicho de otra forma, corresponden a la capacidad de articular y movilizar recursos aprendidos-saberescon vistas a un desempeño de excelencia.

Las competencias se expresan en la acción y suponen la movilización de conocimientos, habilidades y actitudes que la persona ha aprendido en contextos educativos formales e informales. Con la finalidad de promover que alumnos y alumnas desarrollen competencias, el currículum incorpora los conocimientos de las distintas disciplinas que son fundamentales para comprender la realidad, las habilidades cognitivas y procedimientos que posibilitan integrar y movilizar recursos, y las actitudes personales y éticas que orientan una acción responsable consigo mismo y los demás.

Además, asumiendo que las competencias se desarrollan en la práctica y que lo relevante es que alumnos y alumnas logren aprendizajes que puedan transferir a contextos reales, el currículum prioriza la comprensión profunda, el aprendizaje activo, las relaciones entre saberes y la movilización integrada de conocimientos, habilidades y actitudes, en diversos contextos, preferentemente auténticos o reales (MINEDUC, 2009: 10).

Es fácil observar la promoción del desarrollo de competencias superiores. Otro motivo para incluir la cita anterior es para visualizar el hecho que, en teoría, la enseñanza pre-universitaria ya se encuentra alineada - a lo menos en teoría- con el desarrollo de competencias. Según nuestra opinión ambos enfoques son de corte constructivista desde la psicología cognoscitiva.

En particular, la idea del profesor como mediador entre el conocimiento y el aprendiz puede ser encontrado en las teorías desarrollada por Lev Semionovich Vigotsky. Este psicólogo soviético fallecido relativamente joven y que gracias a su hija pudieron conocerse en 1956 sus escritos originados en 1934 y prohibidos en Rusia, enfoca la problemática del desarrollo de una manera distinta a la de las estructuras lógicas o desde las capacidades para procesar la información. Su postura parte del supuesto que el desarrollo y el aprendizaje se influyen mutuamente, creando su teoría de la Zona del Desarrollo Próximo, como consecuencia postula la existencia de dos niveles evolutivos: 
a) El nivel evolutivo real, nivel de desarrollo de funciones mentales del aprendiz, que resulta de ciclos evolutivos cumplidos a cabalidad, en otras palabras, lo que ha conseguido por sí mismo.

b) Un segundo nivel evolutivo potencial, aquello que el aprendiz no puede solucionar por sí solo, pero sí con la ayuda de los demás, por ejemplo un profesor o sus compañeros.

La Zona de Desarrollo Próximo correspondería a la identificación de las funciones que aún no han madurado pera que están en este proceso; según el autor serían los "capullos" en lugar de los "frutos" del desarrollo. En términos prácticos podría considerarse como la distancia del desarrollo entre ambos niveles (Vigotsky, 1988). Naturalmente, la teoría vigotskiana es mucho más extensa y profunda, pero su análisis completo escapa los límites de este trabajo.

El sesgo constructivista involucrado en la expresión la construcción de su propio aprendizaje nos lleva a relacionarlo con la visión de David Ausubel denominada aprendizaje significativo, que según su discípulo Novak (1988) lo expresa como una contraposición al conocimiento memorístico. Un individuo que aprende significativamente debe tratar de relacionar los nuevos conocimientos con los conceptos y proposiciones relevantes que ya conoce. Por el contrario, el llamado aprendizaje memorístico, el nuevo aprendizaje puede adquirirse simplemente mediante la memorización verbal, pudiendo incorporarse arbitrariamente a la estructura de conocimientos (cognitiva) de una persona, sin ninguna interacción con lo que ya existe en ella.

Otro punto relevante de la teoría ausbeliana es el hecho que todos los seres humanos tenemos una estructura cognitiva -a la que le da una gran relevancia en el proceso de aprendizaje- y la entiende como: el conjunto de conceptos e ideas que un individuo posee sobre un determinado campo de conocimientos, así como la forma en la que los tiene organizados.

Este concepto es crucial para el aprendizaje al punto que el proceso de "dictar clases" o enseñar no es otra cosa que el intercambio de estructuras cognitivas entre el que enseña y el que aprende. Novak, ya citado, es taxativo en plantear que el conocimiento y el aprendizaje son cosas distintas, donde el primero es público y compartido, mientras que el segundo es personal e idiosincrático, por lo tanto, nadie aprende si no lo desea de allí es que el aprendizaje debe ser negociado (en palabras de Novak). El concepto negociación del conocimiento en sintonía con la teoría de Ausubel plantea que cuando un nuevo conocimiento pretende penetrar la estructura cognitiva se producen dos fenómenos simultáneos:

a) La diferenciación progresiva: en el curso del aprendizaje significativo, los conceptos que interactúan con el nuevo conocimiento y que sirven de base para la atribución de nuevos significados, van también modificándose en función de esa interacción, o sea van adquiriendo nuevos significados y diferenciándose progresivamente.

b) La reconciliación integrativa: en el curso del aprendizaje significativo se establecen relaciones entre ideas, conceptos, proposiciones ya establecidos en la estructura cognitiva, o sea, relaciones. Los elementos que ya existen en la estructura cognitiva con determinado grado de claridad, estabilidad y diferenciación son percibidos como relacionados, adquieren nuevos significados y llevan a una reorganización de la estruc- 
tura cognitiva. La estructura cognitiva está constantemente reestructurándose durante el aprendizaje significativo.

El proceso es dinámico; el conocimiento va siendo construido. En el aprendizaje significativo, el nuevo conocimiento nunca es internalizado de manera literal — como ya se dijo- porque en el momento en que pasa a tener significado para el aprendiz, entra en escena el componente idiosincrático de la significación. Aprender significativamente implica atribuir significados y éstos siempre tienen componentes personales. El aprendizaje sin atribución de significados personales, sin relación con el conocimiento preexistente, es mecánico, no significativo. En el aprendizaje mecánico el nuevo conocimiento es almacenado de manera arbitraria y literal en la mente del individuo (Moreira, 2000).

Habiendo planteado elementos de reflexión para determinar un marco teórico que sustente el modelo pedagógico de un enfoque enseñanza basado en competencias, pasamos a revisar la problemática de la evaluación que conlleva dicho modelo.

\section{LA EVALUACIÓN DE LA ENSEÑANZA Y APRENDIZAJE DE COMPETENCIAS}

Nuevamente nos encontramos con una polisemia conceptual, para resolverla nos apoyaremos en la postura de Castillo (2002), quien afirma respecto a la evaluación, ya sea en el nivel oficial o en el de autores especializados, subyace una suerte de estructura básica, la que se puede graficar tal como se muestra en la figura siguiente.

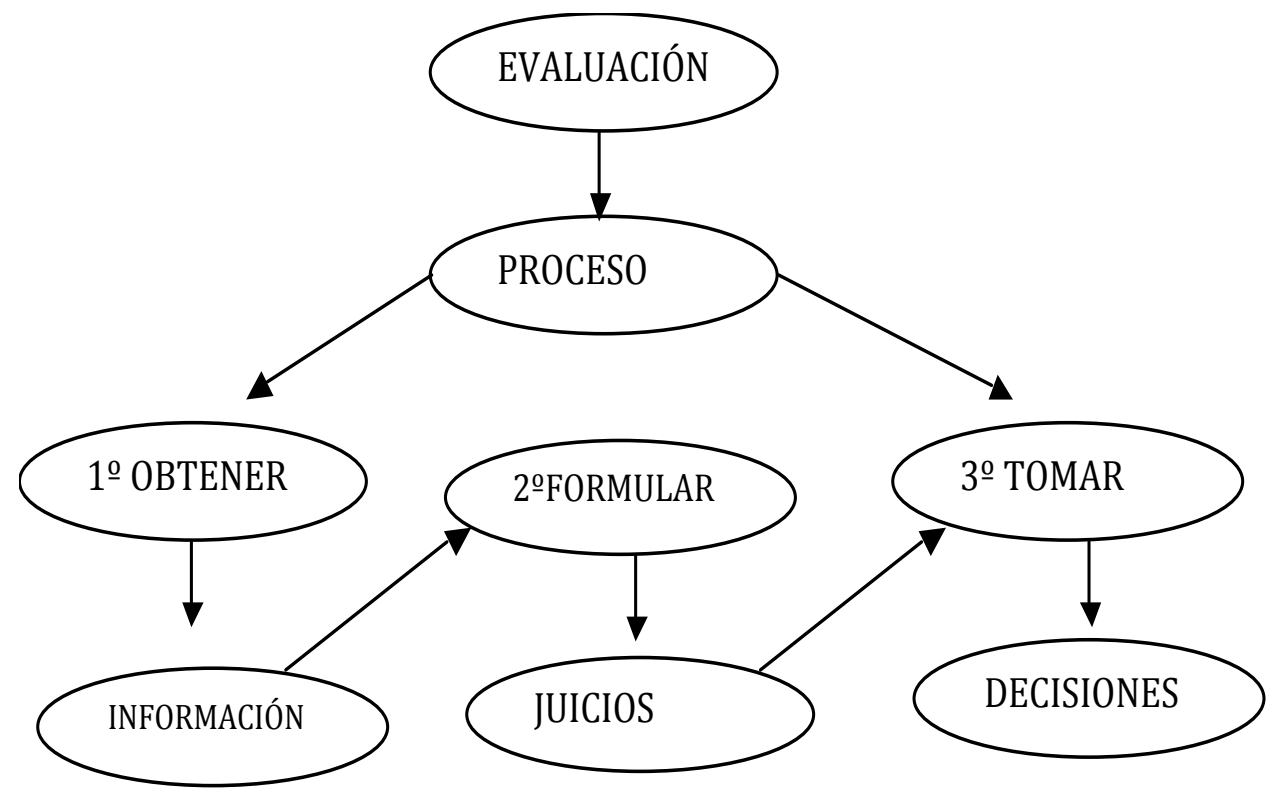


Queda claro que este modelo es aplicable a cualquier componente del acto de enseñanza y aprendizaje (profesor, estudiante, metodología, medios, entidad, etc.). Por otra parte el Ministerio de Educación (MINEDUC) a través de su Unidad de Currículum y Evaluación (UCE) cita a Tenbrink para definir evaluación como: "Evaluación es el proceso de obtener información y usarla para formar juicios que a su vez se utilizarán en la toma de decisiones", absolutamente coincidente con el diagrama (MINEDUC, 2006:33).

La UCE, por su parte, desde varios años propicia lo que llama "Evaluación para el aprendizaje": "Es el proceso de recoger evidencias e información acerca de los aprendizajes logrados por los alumnos y alumnas utilizando criterios de evaluación preestablecidos. El análisis de los resultados es utilizado para retroalimentar con el fin de promover el aprendizaje y realizar los ajustes necesarios en la planificación de actividades en aula" (MINEDUC, 2006:305). Por lo expresado, podemos concluir que el concepto de evaluación debe ser entendido desde dos dimensiones: a) con fines de certificación o calificación el cual es de uso casi exclusivo. b) con fines pedagógicos, que está exactamente en línea con la evaluación para el aprendizaje.

Para finalizar esta parte, recordemos que la didáctica tiene como objetivo: el estudio, la comprensión y mejora de los procesos de aprendizaje y enseñanza. Un acto didáctico tiene lugar cuando se establece una comunicación de doble vía entre docentes y dicentes en función de un contenido determinado. Desde la perspectiva de la evaluación para el aprendizaje la evaluación se torna un elemento crucial, pues origina lo que se llama punto de encuentro didáctico (la intersección de los contenidos, dicente y docente). Esta idea de Castillo (2002) puede resumirse en que la evaluación, en su estado actual, se ha convertido en la clave que facilita la comunicación entre el docente y el alumno, es

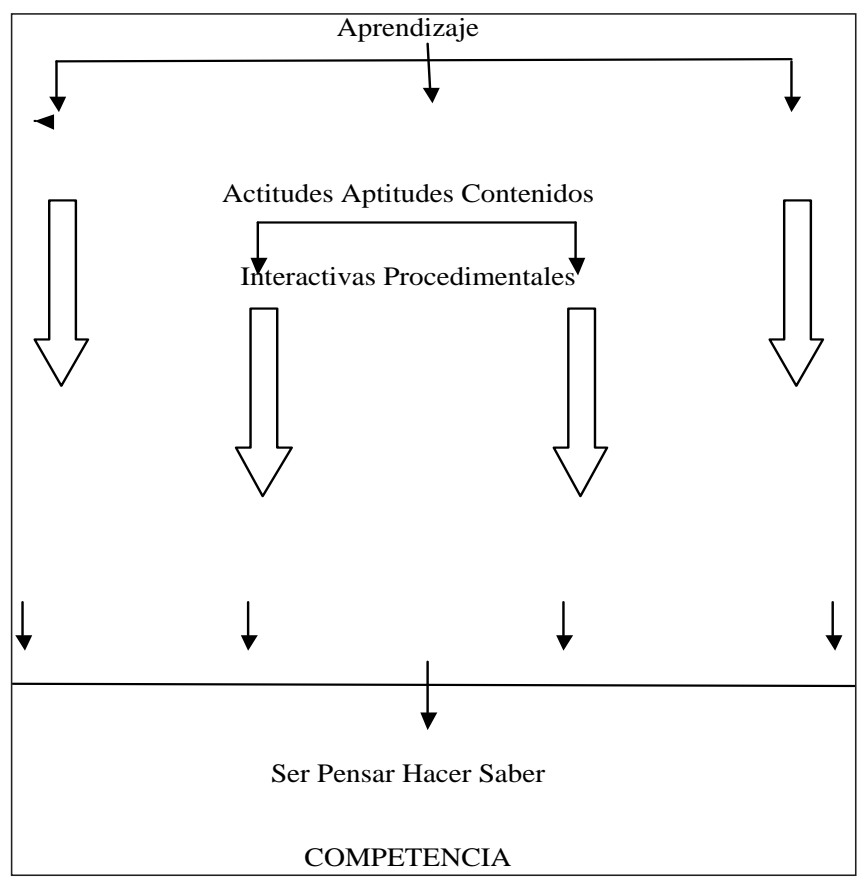

Factores que intervienen en el aprendizaje 
decir, en el vehículo para la aproximación progresiva de las representaciones que sobre los contenidos tienen el aprendiz y su maestro.

Conforme al enfoque ausbeliano el aprendizaje se concibe como la reconstrucción de los esquemas de conocimiento del sujeto a partir de las experiencias que éste tiene con los objetos -interactividad- y con las personas -intersubjetividad- en situaciones de interacción que sean significativas de acuerdo con su nivel de desarrollo y los contextos sociales que le dan sentido. Desde esta perspectiva, el proceso de aprendizaje ocurre de modo que el sujeto del aprendizaje procesa la información de manera sistemática y organizada y no solo de manera memorística, sino que construye conocimiento (Díaz y Fernández 1998). En este proceso se pueden identificar claramente tres factores que son determinantes en el aprendizaje (Iafrancesco, 2004), como son las actitudes, las aptitudes y los contenidos.

Para comprender a cabalidad el diagrama anterior conceptualizaremos los componentes fundamentales que deben ser considerados en el proceso de aprendizaje y enseñanza: la actitud, en el contexto de este trabajo, debe ser entendida como una predisposición afectiva y motivacional requerida para el desarrollo de una determinada acción, posee también un componente cognitivo y un componente comportamental. En la actitud lo fundamental es generar expectativa, porque así el estudiante se interesa y se motiva en su proceso de aprendizaje. No obstante la actitud puede ser inversamente proporcional a la aptitud por un mecanismo de compensación de debilidades, como en el caso de quienes al reconocer sus debilidades en el área de las matemáticas, en medio de la necesidad de aprender, se interesan más por aprender que aquellos que tienen más habilidades para dicha área.

Respecto a las aptitudes intelectivas, éstas corresponden a las habilidades mentales que determinan el potencial de aprendizaje, también definidas como las capacidades para pensar y saber (Iafrancesco, 2004). Dependen de la estructura mental, las funciones cognitivas, los procesos de pensamiento y las inteligencias múltiples. Por su parte, las aptitudes procedimentales son las capacidades para actuar y están relacionadas con los métodos, técnicas, procesos y estrategias empleadas en el desempeño. Finalmente en este esquema, el conocimiento debe ser entendido como toda estructura conceptual susceptible de ser aprendida. Su organización es vital para el proceso de aprendizaje. En la medida en que exista más coherencia entre ellos, los estudiantes encontrarán las relaciones entre los mismos lo que a su vez aumentará su nivel de compresión. La comprensión de los conceptos determina el aprendizaje, más no el aprendizaje significativo; un buen desempeño implica un dominio conceptual añadido a la metacognición lo que conlleva al desarrollo de la competencia.

\subsection{INSTRUMENTOS DE EVALUACIÓN}

Uno de los problemas que plantea la evaluación de competencias es que ellas necesariamente son el producto de un proceso secuenciado; la evaluación debería propender a la constatación del dominio de una competencia, lo que difícilmente puede ser determinada a través de un sólo método. Para fijar ideas, nos referiremos a la llamada Pirámide de Miller, un modelo para evaluar competencias planteado en la década del 90 (Miller, 1990) en el ámbito de la enseñanza de la medicina y que presentamos a continuación: 


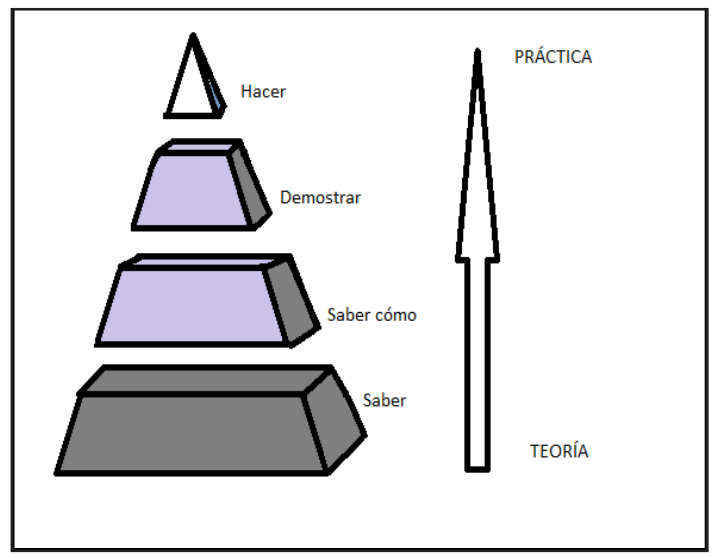

Pirámide de Miller (1990)

La figura muestra con claridad las etapas que debe escalarse para considerar una competencia desarrollada. Las dos primeras etapas (base) están evidentemente relacionadas con lo cognitivo y las dos superiores con el comportamiento.

Es relativamente obvio que los niveles denominados saber y saber como podrían evaluarse a través de instrumentos tradicionales en el contexto de un modelo que intenta certificar el dominio de tópicos tratados por el docente en clases, con énfasis en pruebas de papel y lápiz y que de alguna manera refuerzan el pensamiento convergente. En un enfoque de competencias, esta práctica vendría corresponder al ámbito de lo que se define como capacidad -componente específico que forma parte de una competencia- definida en términos de acciones sobre contenidos ejecutados en torno a tareas que cobran sentido siempre y cuando estén dentro de un contexto de la propia capacidad que se pretenden evidenciar, lo que presenta la restricción de que los constructos en este escenario miden conocimientos genéricos, es decir, apuntarían hacia competencias generales y no necesariamente en un contexto adecuado. Esto último no es menor: supongamos que se está evaluando la tarea "medir una altura"; esta tarea debería estar en el contexto de una acción que estuviera conectada con una competencia profesional, más allá de la aplicación experimental de método científico en general.

Si aceptamos la validez de la pirámide de Miller, el proceso de evaluación de competencias se presenta como algo muy complejo en todas sus etapas. Nuestra breve discusión desarrollada en el párrafo anterior evidencia la necesidad de un cambio cultural extremadamente profundo tanto en docentes y dicentes cuya discusión, creemos, es una tarea urgente y aún pendiente.

Como un pequeño aporte para sortear los problemas que aquí se han planteado discutiremos tres recursos evaluativos que según nuestra opinión estarían alineados con recursos metodológicos apropiados para medir el desarrollo de competencias.

\subsubsection{Los mapas conceptuales}

En la teoría ausbeliana los conocimientos previos -lo que el o la estudiante ya sabe sobre un nuevo conocimiento- ocupa un lugar central en el proceso del aprendizaje 
significativo, pues estos pueden bloquear o potenciar el procesamiento adecuado de la nueva información en nuestra estructura cognitiva. Para evaluar el estado de los conocimientos previos Novak y Gowin (1988) crearon los llamados Mapas Conceptuales, representaciones mentales de conceptos ampliamente aceptados y mencionado en la literatura especializada. El mapeamiento conceptual es una técnica muy flexible que puede ser usada en diversas situaciones para diferentes finalidades: instrumento de análisis del currículum, técnica didáctica, recurso de aprendizaje, medio de evaluación (Moreira 2000).

Es posible trazar un mapa para una única clase, para una unidad de estudio, para un curso y hasta para un programa educacional completo. La diferencia está en el grado de generalización e inclusión de los conceptos colocados en el mapa. Un mapa que envuelva apenas conceptos generales, inclusivos y organizacionales puede ser usado como referencial para la planificación de un curso entero, mientras que un mapa que sólo incluya conceptos específicos, poco inclusivos, puede ayudar en la selección de determinados materiales instruccionales. Esto significa que los mapas conceptuales pueden ser importantes mecanismos para focalizar la atención del planificador del currículum en la distinción entre el contenido que se espera que sea aprendido y aquel que sirve de vehículo de aprendizaje. El contenido curricular está contenido en fuentes de conocimiento tales como artículos de investigación, ensayos, poemas, libros. Los mapas conceptuales pueden ser útiles en el análisis de esos documentos con la finalidad de adecuar para la instrucción el conocimiento contenido en ellos. Se considera aquí que el currículo se refiere al conjunto de conocimientos. Así, el análisis de la estructura del conocimiento implica el análisis del currículum y el mapeamiento conceptual puede ser un instrumento útil en ese análisis.

De manera análoga, los mapas conceptuales pueden usarse para mostrar relaciones significativas entre los conceptos enseñados en una sola clase, en una unidad de estudio o en un curso entero. Son representaciones concisas de las estructuras conceptuales que están siendo enseñadas y como tal, probablemente facilitan el aprendizaje de esas estructuras. Sin embargo, a diferencia de otros materiales didácticos, los mapas conceptuales no son auto-instructivos: deben ser explicados por el profesor. Además, aunque puedan usarse para dar una visión general del tema en estudio es preferible usarlos cuando los alumnos ya tienen una cierta familiaridad en el asunto, de modo que sean potencialmente significativos y permitan la integración, reconciliación y diferenciación de significados de conceptos (Morira 2000). En la medida en que los alumnos utilicen mapas conceptuales para integrar, reconciliar y diferenciar conceptos; en la medida en que usen esa técnica para analizar artículos, textos, capítulos de libros, novelas, experimentos de laboratorio y otros materiales educativos del currículum, estarán usando el mapeamiento conceptual como un recurso de aprendizaje.

Como instrumento de evaluación del aprendizaje, los mapas conceptuales pueden utilizarse para obtener una visualización de la organización conceptual que el aprendiz atribuye a un determinado conocimiento. Se trata básicamente de una técnica no tradicional de evaluación que busca informaciones sobre los significados y relaciones significativas entre conceptos claves de la materia de enseñanza desde el punto de vista del alumno.

Finalmente, dejamos constancia que hay un sitio especializado, para trazar y compartir mapas conceptuales patrocinado por el Institute for Human and Machine Cognition (http://cmap.ihmc.us/), supervisado por el propio Novak. 


\subsubsection{Matrices de valoración o rúbricas}

Otro componente inherente a la evaluación de competencias es que estas deben expresarse en indicadores o comportamientos que puedan observarse de alguna manera, una posible alternativa para ello es recurrir a una tabla de doble entrada, también denominada rúbrica que según Herrera (2001) es una pauta, minuta o tabla que nos permite aunar criterios de evaluación, niveles de logro y descriptores. Es un recurso lo suficientemente flexible que se puede regular en la práctica hasta encontrar el justo valor de la evaluación que hacemos. También es muy importante su carácter de pre-establecida, acordada y socializada con los alumnos y alumnas, antes de aplicar la evaluación. Tiene la propiedad de un instrumento descriptor de medición cualitativo que establece criterios, estándares y la naturaleza de un desempeño, donde se debe entender como indicador: aquello que se acepta como evidencia del estado actual de un objeto, sujeto, programa, proceso o producto, con respecto a un criterio dado. Consecuentemente, un estándar correspondería a un punto de referencia que se considera para determinar la aceptación o excelencia de algo; define la calidad de algo para ser considerado adecuado o inadecuado y proporciona orientaciones para interpretar los resultados de una evaluación (Conde y Pozuelo, 2007). También existe una página especializada para generar y compartir rúbricas (http:// rubistar.4teachers.org/index.php?\&skin=es\&lang=es\&).

\subsubsection{Portafolios}

El portafolio o carpeta consiste en un archivador que incluye todo lo que hace el alumno, tal como: apuntes o notas de clases, trabajos de investigación, guías de trabajo y su desarrollo, comentarios de notas, resúmenes, pruebas escritas, autoevaluaciones, tareas desarrolladas, comentarios de progreso del alumno realizado por el profesor, los cuales son ordenados según determinados criterios o características de las actividades de aprendizaje (Barrios, 1998).

En opinión de Monzó (2006), una evaluación basada en competencias requiere que aquellos que están siendo evaluados, demuestren, construyan, desarrollen un producto o solución a partir de unas condiciones y estándares definidos, en un momento determinado. En este sentido, la utilización de los portafolios implica que los alumnos, profesionales o personas que buscan su primer empleo se vean obligados a estructurar las tareas por las que van a ser evaluados, aplicar sus conocimientos previos, elaborar sus respuestas e, incluso, explicar el proceso que les ha llevado a las mismas. Esta autora distingue tres tipos de portafolios, dependiendo de su intención:

a) Portafolios de enseñanza, referido a la actuación docente.

b) Portafolios de aprendizaje, entendido como una estrategia de adquisición de conocimientos.

e) Portafolios profesionales, relacionado con los procesos de inserción o reinserción profesional y social.

Entre sus principales ventajas podría señalarse que permite al profesor aplicar en forma activa y directa la evaluación formativa o de proceso, como un proceso interactivo: alumno-portafolio-profesor, cuya interacción y comunicación se basa en los materiales elaborados por los alumnos, que permiten detectar o inferir sus logros parciales o terminales con relación a determinados objetivos educacionales. 
$\mathrm{Su}$ estructura, una acumulación ordenada por secciones, debidamente identificadas o etiquetadas que contienen los registros o materiales productos de las actividades de aprendizaje realizadas por el alumno en un período de tiempo, con los comentarios y calificaciones asignadas por el profesor, permite visualizar el progreso del alumno en ese período de tiempo. Además, facilita la autoevaluación del trabajo realizado por parte del alumno, como tomar conciencia de sus logros y las relaciones de estos con su entorno mediato, por tanto, es un medio que permite integrar activamente la enseñanza y la evaluación en forma directa, como facilitar la comunicación entre alumno y profesor.

Las características de un portafolio de trabajo pueden ser determinadas por cada profesor e incluso orientar al alumno acerca de las secciones que debe contener, además cada alumno podrá diseñar en forma libre la identificación de cada sección y la forma de insertar los distintos materiales.

\section{A MODO DE CIERRE}

Hemos desarrollado un rápido recorrido por las fronteras del mundo de las competencias, evitando hasta donde fue posible no caer en una suerte de reduccionismo que pudiese contaminar la discusión de una materia extremadamente compleja.

Es evidente que nuestro análisis curricular lo hemos enfocado desde un sesgo constructivista, lo que sin duda es absolutamente discutible, pero para ello se tuvo en vista que nuestra intención primordial es el de alentar un debate sobre el tema didáctico respecto al desarrollo de las competencias en general y de la evaluación de competencias en particular. Por todo lo expresado, debiera quedar de manifiesto la necesidad previa de optar por uno entre varios enfoques curriculares coherente con el desarrollo de competencias. A este respecto Ramírez y Medina (2008) resaltan los aportes de los dos enfoques de mayor aceptación en la actualidad:

a) El enfoque conductista y su promesa que se puede observar y demostrar a lo largo de un proceso formativo el grado que se han logrado las competencias.

b) Por su parte el enfoque constructivista de la educación basada en competencias, extiende la necesidad de lograr en los estudiantes la transferencia de los conocimientos no sólo a contexto inmediatos, sino a la vida misma, a lo que viven los estudiantes aquí y ahora, y también a lo que tal vez necesiten para poder potenciar su vida futura. Así se plantean contenidos que tienen significado integral para la vida.

Es evidente que el enfoque en competencias debería modificar los puntos de vista convencionales de la forma que se aprende y enseña, pues a esta altura de la discusión, debería estar claro que el punto central no es la acumulación de conocimientos, sino el desarrollo de las posibilidades que pose cualquier individuo de recorrer la mencionada pirámide de Miller.

Lo que nos lleva al nudo central de nuestro trabajo, el presentar elementos de una discusión pendiente en el ámbito académico, el salto cuántico intelectual que se debe producir para que se implemente un proceso evaluativo que realmente mensure el logro de competencias. 


\section{REFERENCIAS BIBLIOGRÁFICAS}

Ausubel, D. N. (1983). Psicología Educativa: Un punto de vista cognoscitivo. México: Trillas.

Barrios, O. (1998). Uso del portafolios del alumno. Santiago: UMCE.

Beneitone, P. , Esquetini,C., González, J., Marty, M., Siufi, G. y Wagenaar, R. (2007). Reflexiones y perspectivas de la Educación Superior en America Latina. Bilbao: Publicaciones de la Universidad de Deusto.

Brunner, J. (2003). Educación e Internet: ¿La Próxima Revolución? Santiago: Fondo de Cultura Económica.

Cabrerizo, J., Rubio, N., y Castillo, S. (2008). Programación por competencias. Madrid: Pearson.

Castillo, S. (2002). Compromisos de la Evaluación Educativa. Madrid: Pentrice Hall.

Conde, A. y Pozuelo, F. (2007). Las plantillas de evaluación (rubrica) como Instrumento para la evaluación. Un estudio de caso en el marco de la reforma de la enseñanza universitaria en el EEES. Investigación en la Escuela, n. 63, 77-90.

Diaz, F. y Hernández, G. (1998). Estrategias docentes para un aprendizaje significativo. México: Mc Graw.

Herrera, R. (2001). Evaluación de los Aprendizajes Escolares. CPEIP: Santiago.

Ianfrancesco, G. (2004). La evaluación integral y del Aprendizaje: Fundamentos y estrategias. Bogotá: Cooperativa Editarial Magisterio.

Miller, G. (1990). The assessment of Clinical Skill. Compotence-Performance. Academic Medicine, vol. 9 , n. $65,63-67$.

MINEDUC. (2006). Evaluación para el Aprendizaje. Santiago: Unidad de Currículum y Evaluación.

MINEDUC. (2009). Objetivos Fundamentales y Contenidos Mínimos Obligatorios de la educación Básica y Media. Actualización 2009. Santiago: Ministerio de Educación, República de Chile.

Monzó, R. (2006). Concepto de Evaluación en la Evaluación Educativa. México D. F.: Publicaciones Cruz O., S.A.

Moreira, M. (2000). Aprendizaje Significativo: teoría y práctica. Madrid: Visor.

Muñoz, A. y Sobrero, V. (2006). Proyecto Tuning en Chile: Análisis de Procesos de Internalización de la Educación Superior. Calidad de la Educación, vol. 24, 249-271. Visitado el 26 de septiembre 2010 en http://www.cned.cl/public/secciones/seccionpublicaciones/doc/.

Novak, J. y Gowin, D. (1988). Aprendiendo a aprender. Barcelona: Martínez Roca.

Ramírez, L. yMedina, M. (2008). Educación basada en competencias y el proyecto Tuning en Europa y Latino América. Su impacto en México. Ide@s CONCYTENG, n. 39, 97-111.Visitado el 26 de septiembre de 2010 en http://octi.guanajuato.gob.mx

Tenbrink, T. (1984). Evaluación: guía práctica para profesores. Madrid: Narcea.

Vigotsky, L. (1988). El desarrollo de los proceso psicológicos superiores. México: Crítica Grijalbo. 